\title{
PRESTRESS TUNING OF THE NONLINEAR DYNAMICS OF TENSEGRITY METAMATERIALS
}

\author{
F. Fraternali ${ }^{1}$, A. Amendola ${ }^{1}$, E. Hernández-Nava ${ }^{2}$, R. Goodall ${ }^{2}$, R.E. Skelton ${ }^{3}$ and V.F. \\ Nesterenko ${ }^{3}$ \\ ${ }^{1}$ Department of Civil Engineering, University of Salerno, 84084, Fisciano (SA), Italy \\ e-mail: \{f.fraternali, adaamendola1\}@unisa.it \\ (A. Amendola, F. Fraternali) \\ ${ }^{2}$ Department of Materials Science and Engineering, University of Sheffield, Mappin Street, Sheffield, \\ S1 3JD, UK \\ e-mail: \{mtq10eh, r.goodall $\} @$ sheffield.ac.uk \\ (E. Hernández-Nava, R. Goodall) \\ ${ }^{3}$ University of California San Diego, MAE/Aero, 9500 Gilman Dr., La Jolla, CA 92093, USA \\ e-mail: \{bobskelton, vnesterenko\}@ucsd.edu \\ (R.E. Skelton, V.F. Nesterenko)
}

Keywords: Tensegrity Prisms, Additive Manufacturing, Electron Beam Melting, Nonlinear Response, Mechanical metamaterials.

\begin{abstract}
We investigate the experimental response of additively manufactured tensegrity prisms under compression loading and different states of internal prestress. Physical models of tensegrity prisms are additively manufactured through an electron beam melting facility. Spectra strings are applied to the 3D printed models through a post-tensioning approach, which endows the structure with the desired prestress. Experimental tests highlight that the examined structures exhibit strongly nonlinear mechanical response under compression, which appears to be useful for the construction of a new class of nonlinear mechanical metamaterials featuring solitary wave dynamics to be controlled by internal and external prestress.
\end{abstract}




\section{INTRODUCTION}

One of the most common techniques nowadays employed for the fabrication and the rapid prototyping of innovative periodic structures and lattice materials is additive manufacturing (AM). Several fabrication methods have been proposed in this field, with resolution ranging from the centimetre- to the nanometre-scale. It is worth mentioning polyjet $3 \mathrm{~d}$ printing technologies; Electron Beam Melting (EBM); x-ray lithography; deep UltraViolet lithography; soft lithography; two-photon polymerization; atomic layer deposition; and projection microstereolitography, among other available methods (refer to [1]-[5], and references therein). The processing of detailed components through the consecutive addition of small quantities of material, usually in thinner layer than $100 \mu \mathrm{m}$, makes AM capable of producing highly complex structures at different scales. EBM is a popular AM technique used to fabricate metallic structures. Its name is down to the technique's deposition, in which, it makes use of a beam of electrons to fully melt powder particles of a conductive material. This melting operation is performed in a selective way to produce a structure corresponding to a surface file, commonly STL. The use of EBM has been successfully demonstrated in several studies of structured materials including; lattices with graded porosity [4], metallic foams [6], optimized topologies [7] and structures with negative Poisson's ratio [8] to name a few.

Tensegrity structures are axially loaded prestressable structures. Motivated by nature, where tensegrity concepts recurrently appear [9], engineers have only recently developed efficient analytical methods to exploit tensegrity concepts in engineering design. Traditional construction of tensegrity structures through manually assembling of different components can represent a challenging task. Potentially resulting in a series of mitigated results because of structural and connecting issues; eccentricities at the joints, unwanted movements and friction (refer, e.g., to [10] and references therein). Hence in order to systematically study complex tensegrity systems, the development of fabrication techniques based on AM would be of great help. The paper begins by describing the Computer Aided Design (CAD) of the tensegrity prisms to be manufactured, which consist of three titanium alloy (Ti6Al4V) bars; two Ti6Al4V plates (bases), and three Spectra fibers connecting the base plates (Sect. 2). Sect. 3 describes the EBM process used for the AM manufacturing of temporary models without the Spectra fibers, while Sect. 4 illustrates the placement and the post-tensioning of such elements within the final models. The experimental part of the study presents quasi-static compression tests that investigate on the response of the examined structures under compressive axial loading. The given results validate the theoretical predictions presented in [10,11], highlighting the geometrically non-linear nature of the axial force vs. axial displacement response of such structures (Sect. 5). Sect. 6 illustrates the wave dynamics of chains of tensegrity prisms featuring either hardening-type or softening-type nonlinear response, highlighting that hardening-type chains support compression solitary waves, while softening-type chains support rarefaction solitary waves. We end in Sect. 7, by drawing the main conclusions of the present study and future research lines.

\section{GEOMETRIC MODELS OF TENSEGRITY PRISMS}

We focus in the present Section on the computer aided design of provisional models of structures showing tensegrity prisms endowed with rigid bases as building blocks [10]. Each prism is composed of three struts, two rigid bases (a top-base, and a bottom-base), and three cross-cables. Fig. 1 shows a computer model of metallic structures formed by the struts and the terminal bases of the building blocks, plus some sacrificial supports connecting the bases of the building blocks (vertical elements in Figs. 1). The connections between the bars and the 
bases are realized through hemispherical joints (cf. Fig. 1). Such provisional structures will be additively manufactured in a titanium alloy (Ti6Al4V) through the EBM facility Arcam S12 (cf. Sect. 3). Next, the 3D printed structures will be finished with the addition of tensioned Spectra strings connecting the struts and the terminal bases of the building blocks. Once the insertion of the Spectra strings is completed, and a suitable prestress is applied to the structure, the sacrificial supports will be removed (post-tensioning approach, cf. Sect.4). We will now show in detail how we design the building blocks of the structures under investigation. To ensure zero bending moments, the extremities of the bars composing such units should have almost zero diameter at the nodes. Unfortunately, it is impossible to additively manufacture elements with nearly zero diameter, due to the limited precision tolerance of the available printing equipment. In the present case the ARCAM S12 EBM facility allows to smoothly manufacture features with size down to $0.5 \mathrm{~mm}$ [6]. Such a limitation also affects the manufacturing of the tensile elements (cables or strings) of the building blocks, due to the small diameter that is needed for such elements. As already explained, our fabrication strategy makes use of 3D printed partially complete models in which the strings are replaced by sacrificial supports with diameter $d^{*}=0.5$ $\mathrm{mm}$. The latter are removed in a second phase, when Spectra strings are manually inserted into the building blocks, with the aim of 'sewing' the 3D printed models (Sect.4). To this end, the models to be 3D printed show holes passing through the joints, which will host the Spectra strings in the post-tensioning phase. We design the compressive elements (bars or struts) of the temporary models with a bi-conic shape obtained by joining two truncated cones. The latter feature decreasing radii towards the extremities (Fig. 1), so as to minimize the bending rigidity of the nodes. Let $d$ denote the minimum diameter of the struts (at the extremities), and let $D$ denote the maximum diameter of such elements (at the mid-span). Following [12] we make use of the following tapering ratio: $\beta=D / d=6$. Overall, each building block to be 3D printed is composed of three truncated bi-cones (bars), three sacrificial supports, two triangular plates of thickness $t$ (bases), and six hemispherical joints with radius $r$ (Fig. 1). Table 1 shows the geometric properties of such elements, making use of the symbols $s_{N}, b_{N}$ and $h_{N}$ to respectively denote the length of the edges of the base plates; the length of the struts; and the height of the prism (measured from the centre to centre of the bases), in correspondence with the unstressed (or natural) configuration of the structure.

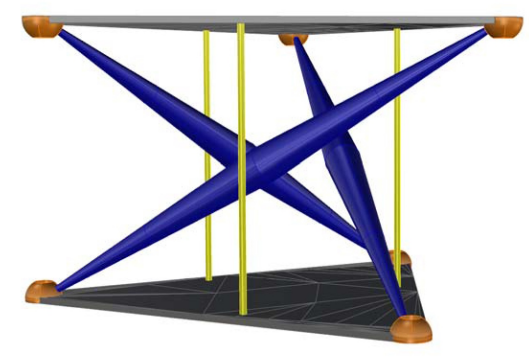

Figure 1: Graphical model of a building block composed of three sacrificial supports (yellow), six hemispherical joints (orange), three struts (blue) and two terminal plates (gray). 


\begin{tabular}{|c|c|c|c|c|c|c|c|}
\hline$t(\mathrm{~mm})$ & $r(\mathrm{~mm})$ & $s_{N}(\mathrm{~mm})$ & $b_{N}(\mathrm{~mm})$ & $D(\mathrm{~mm})$ & $d(\mathrm{~mm})$ & $d^{*}(\mathrm{~mm})$ & $h_{N}(\mathrm{~mm})$ \\
\hline 1.0 & 1.5 & 34.0 & 44.1 & 3.0 & 0.5 & 0.5 & 22.3 \\
\hline
\end{tabular}

Table 1: Geometrical properties of the building blocks of the examined models.

\section{ADDITIVE MANIFACTURING OF PHYSICAL MODELS}

The present section focuses on the EBM technique employed to process the CAD model illustrated in the previous section. We begin by describing the geometrical and chemical characteristics of the employed Ti6Al4V alloy. Next, we briefly describe the employed manufacturing process. We end by presenting an experimental study on the influence of the direction in which the part is built (i.e. how the layers are built up) on the mechanical properties of the melted Ti-6Al-4V alloy.

\subsection{Raw materials}

The manufacturing process examined in the present work starts with the spreading out of a layer of Ti4Al6V prealloyed powder into the build platform of the printing machine (Arcam EBM S12). Such a powder is provided by the manufacturer and is made of spherical particles with $45-100 \mu \mathrm{m}$ diameter. The raw material is grade 5 under the standards of the American Society of Testing and Materials (ASTM Grade 5, 6Al-4V). It must be considered, however, that the continuous material reuse, which is a common practice in the EBM manufacturing, might slightly change the chemical composition of the Ti6Al4V powder (for example by gradual pick up of oxygen). Tab. 2 provides the chemical composition experimentally determined for the powder employed in the previous study.

\begin{tabular}{ccccccc}
\hline $\mathrm{Ti}$ & $\mathrm{Al}$ & $\mathrm{V}$ & $\mathrm{Fe}$ & $\mathrm{C}$ & $\mathrm{N}$ & $\mathrm{O}$ \\
\hline 88.28 & 6.88 & 4.27 & 0.18 & 0.007 & 0.026 & 0.33
\end{tabular}

Table 2: Chemical composition of the Ti-6Al-4V prealloyed powder in wt $\%$.

\subsection{Manifacturing process}

EBM works in vacuum conditions at $1.9 \times 10^{-3}$ mbar, and heats the base of the build area up to the temperature of $720^{\circ} \mathrm{C}$. Layers of Ti-6Al-4V powder are progressively deposited, heated and melted, according to a sliced version of the CAD model to be manufactured, which is prepared by an internal preprocessor of the Arcam EBM S12. Layer heating (using a defocused beam) is employed to reduce the energy needed for the melting phase. The EBM working parameters are varied for the bars, joints and plates, with the aim of ensuring uniform material density throughout the model, as much as possible (cf. Tab, 3). The voltage is kept constant at $60 \mathrm{kV}$. Once manufactured, all remnants of unmelted powder are removed using compressed air.

\subsection{Parent material properties}

We printed twelve tensegrity prisms with two different building orientations for material characterization purposes. The main goal of such a study was to detect the influence of the EBM build direction on the mechanical properties of the melted Ti-6Al-4V alloy. We built six samples along the vertical direction (V specimens: prism bases parallel to the building plate), and six other samples along the horizontal direction (H specimens: prism bases perpendicular to the building plate). Parts of such specimens, manually cut using shears, were subject to tensile 


\begin{tabular}{cccc}
\hline & $\begin{array}{c}\text { Beam current } \\
(\mathrm{mA})\end{array}$ & $\begin{array}{c}\text { Beam speed } \\
(\mathrm{mm} / \mathrm{s})\end{array}$ & Focus Offset \\
\hline Preheat & 30 & 14600 & 50 \\
Plates & 17 & 500 & 19 \\
Spheres & 1.7 & 200 & 0 \\
Bars & 1.7 & 200 & 0
\end{tabular}

Table 3: Beam parameters employed for the manufacturing of the model parts.

tests according to the ASTM standard E8-13a [13]. The data obtained from tensile tests include the $0.2 \%$ Yield strength (YS), ultimate tensile strength (UTS), and Young's modulus (YM), see Table 3.3. The yield strength of $\mathrm{V}$ - and $\mathrm{H}$-specimens was found to be slightly different (V-specimens more resistant than H-specimens, cf. Table 3.3), hought to be due to changes in material porosity with the deposition angle [14]. It is worth mentioning that the reproducibility of material properties in the AM of lattice materials in general is subject of debate. This is as the fraction of defects, such as internal porosity and surface roughness, dramatically affect the material strength, especially in the case of small features, such as, e.g., the nodal junctions [6]. The results presented in Table 3.3 led us to prefer the vertical deposition technique for the manufacturing of the final specimens (Fig. 2).

\begin{tabular}{cccc}
\hline Build orientation & $0.2 \%$ Y.S. & UTS & Young's modulus \\
\hline & $(\mathrm{MPa})$ & $(\mathrm{MPa})$ & $(\mathrm{GPa})$ \\
Horizontal & $881 \pm 8.4$ & $1040 \pm 6.7$ & $110 \pm 2.1$ \\
Vertical & $923 \pm 10.7$ & $1042 \pm 9.2$ & $116 \pm 2.8$
\end{tabular}

Table 4: Bulk material properties based on tensile tests for two build orientations.

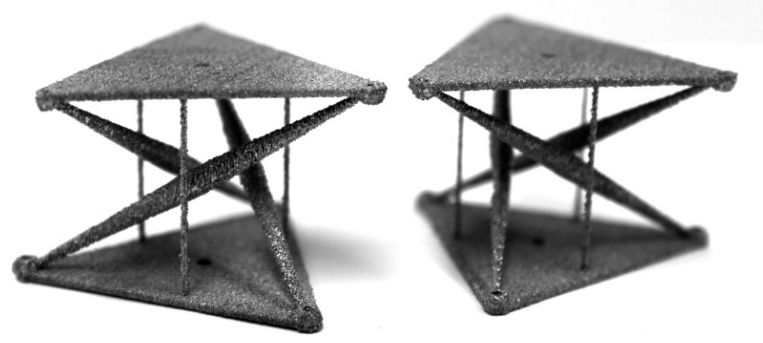

Figure 2: EBM-printed left-handed (left) and right-handed (right) tensegrity prisms.

\section{POST-TENSIONING OF 3D-PRINTED SPECIMENS}

As anticipated, we 'sewed' the 3D printed structures by slipping Spectra ${ }^{\circledR}$ fibers with 0.1 $\mathrm{mm}$ diameter through the holes crossing the joints. We used Spectra fibers supplied by Shimano American Corporation (Irvine, CA, USA), with declared maximum load bearing capacity of 5 $\mathrm{kg}$. Hereafter, we assume that such string elements have Young modulus $E_{s}=5.48 G P a$, based on the results of the experimental study presented in [10]. Since the cross-section area of the strings is $A_{s}=\pi 0.1^{2} / 4=0.0078 \mathrm{~mm}^{2}$, their axial stiffness is computed as: $E_{s} A_{s}=0.043 \mathrm{kN}$. 
After slipping the Spectra strings through the holes crossing the joints, we fixed them in correspondence with the top-base of the structure through slipknots (Fig. 3). Next, we removed the sacrificial supports of the 3D printed structure (cf. Figs. 2, ), and we tensioned the strings by fixing them to the bottom-base. A level was placed on the top-base of the structure during the post-tensioning phase, in order to ensure that such a base was horizontal, and the strings approximatively carried equal tensile forces. We applied the post-tension approach to manufacture single prisms, either left-handed (L-prisms, Fig. 3-left) or right-handed (R-prisms, Fig. 3-right).

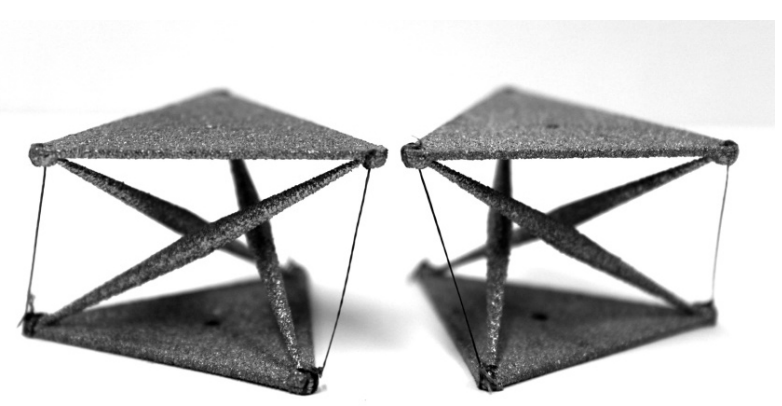

Figure 3: L-prims (left) and R-prism (right) prisms obtained by 'sewing' the EBM-printed models with Spectra strings.

\section{QUASI-STATIC COMPRESSION TESTS}

We investigated on the experimental response of the tensegrity prisms illustrated in the previous section under quasi-static compression loading, on employing a strain rate of $1 \cdot 10^{3} s^{1}$. The testing apparatus consisted of a Zwick/Roell Z050 testing machine equipped with $20 \mathrm{kN}$ load cell (Fig.4). To minimize the frictional effects between the terminal bases of the prisms and the testing machine plates, we carefully lubricated all such surfaces, before initiating the compression tests. We lubricated the joints of the tested structures as well, in order to reduce the friction between the strings and the rough internal surface of the joints.

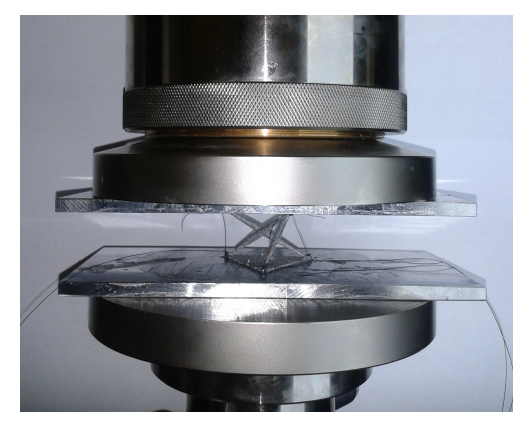

Figure 4: Experimental set-up for the compression testing of post-tensioned structures.

Fig. 5 shows the results of compression tests on different L-prisms and R-prisms, which differ from each other for the value of the applied prestress. The cross-string prestrain $p$ [11] was estimated by comparing the lengths of the cross-strings in the unstressed and prestressed configurations (cf. Fig. 5). The axial force $F$ vs. axial-displacement $\delta$ plots in Fig. 5 highlight 
a marked hardening behavior of the L- and R-prisms (tangent stiffness increasing with $\delta$ ), up to the specimen failure. Such an elastic hardening (or hardening) effect is magnified by increasing the internal prestress (cf. Fig. 5), in agreement with the theoretical results presented in [11] for tensegrity prisms endowed with rigid bases. The specimen collapse was due to the detachment of one or more bars from the joints. It is worth mentioning that we did not observe plastic yielding of the strings or buckling of the bars up to the specimen failure.

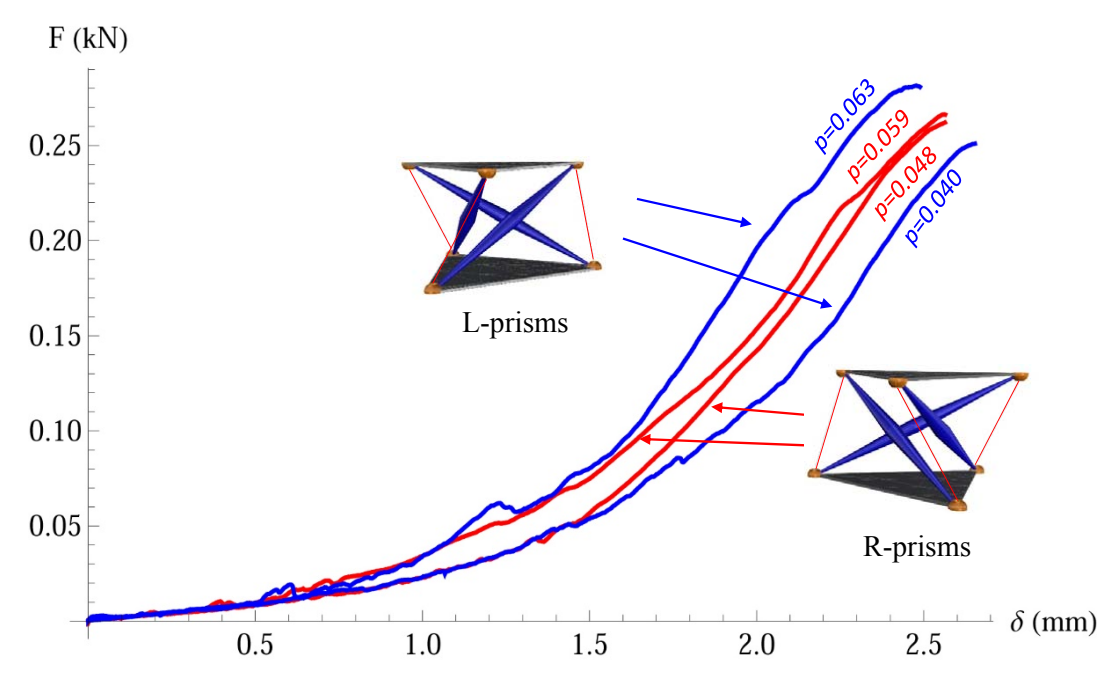

Figure 5: Axial force $F$ vs. axial-displacement $\delta$ curves of single-prism structures under axial compression loads.

\section{NONLINEAR DYNAMICS OF TENSEGRITY METAMATERIALS}

Ordinary engineering materials typically exhibit either elastic hardening (e.g., crystalline solids), or elastic softening (e.g., foams). More puzzling is the geometrically nonlinear response of structural lattices based on tensegrity prisms, which may gradually change their elastic response from hardening to softening through the modification of mechanical, geometrical, and prestress variables. Recent studies have indeed shown that the elastically hardening regime observed in the tensegrity prisms analyzed in Fig. 5 can switch to an elastically softening regime when the rigid bases are replaced with deformable string networks, and the prism features suitable aspect ratios between the height and the radius of the circumference circumscribed to the base triangles $[10,11,19]$. In particular, it has been shown that a low acoustic impedance chain of prisms with with elastic hardening response (LIEH chain) supports compression solitary waves, while a high acoustic impedance chain of prisms with elastic softening response (HIES chain) supports compression solitary waves [19].

Fig. 6-top illustrates the behavior of a compression solitary pulse approaching the interface between two chains in tensionless contact with each other: a LIEH branch with 700 masses and a HIES branch with 2200 masses (refer to [19] for numerical data). The two branches are subjected to different global prestrains $\varepsilon_{s_{0}}$ (initial axial strains of the prisms composing the branches) and local prestrains $p_{0} \equiv p$ (cross-string prestrains). Fig. 6-top shows the evolution of strain pulses in the examined system under the impact velocity $v_{0}=5.0 \mathrm{~m} / \mathrm{s}$. We observe that initial solitary compression wave travels along the LIEH branch. The interaction of such waves 
with the LIEH-HIES interface generates a gap between the branches and unexpected transmitted solitary rarefaction waves with oscillatory tail in the HIES branch. A train of reflected solitary compression waves is observed in the LIEH branch [19]. The results in Fig. 6-bottom instead deal with the interaction of a rarefaction solitary wave with an interface between a HIES lattice of 5000 masses and LIEH lattice of 1000 masses under the the impact velocity $v_{0}=5.0 \mathrm{~m} / \mathrm{s}$ (refer to [19] for numerical data). It is seen that HIES system supports an incident solitary rarefaction pulse with oscillatory tail. Its interaction with the given interface results in a train of transmitted solitary compression waves in LIEH system. A reflected solitary rarefaction wave followed by oscillatory tail propagates back into the HIES branch. The results in Fig. 6-bottom suggest that the oscillatory tail of the incident rarefaction pulse degenerates into an infinitely small amplitude trail.

\section{CONCLUSIONS}

We have investigated on the fabrication of physical models of tensegrity prisms, by combining the additive manufacturing of metallic models, via EBM, with the manual insertion of tensioned Spectra strings in 3D printed structures. Compression tests have been performed with the aim of characterizing the nature of the mechanical response of such structures under large or moderately large axial displacements. We have observed a markedly nonlinear response of the examined structures, and elastic hardening effects under large axial displacements. Such results confirm the outcomes of previous theoretical and experimental studies available in the literature on the mechanical response of tensegrity prisms endowed with rigid bases $[15,10,11]$.

Elastically hardening mechanical metamaterials support compressive solitary waves and the unusual reflection of waves on material interfaces [16]-[19] (cf. Fig. 6). Solitary wave dynamics has been proven to be useful for the construction of a variety of novel acoustic devices. These include: acoustic band gap materials; shock protector devices; acoustic lenses; and energy trapping containers, to name some examples (refer, e.g., to [20] and references therein). The present study represents a first step towards the additive manufacturing and testing of nonlinear tensegrity metatamerials to be employed for real-life engineering devices. We address the formulation and implementation of innovative, multimaterial deposition techniques that are able to apply internal prestress in AM-fabricated tensegrity structures to future work. We plan to fabricate macro- and small-scale models of such structures using materials with different coefficients of thermal expansion for struts and cables. In addition, we plan to manufacture micro-scale tensegrity models through projection micro-stereolitography [5], employing swelling materials for the tensile members [21], and accounting for material fracture [22]. Once dried, the tensile members will contract, creating internal prestress. Engineering applications of the such metamaterials will deal with novel, tunable focus acoustic lenses, impact mitigation devices, bio-inspired membranes and innovative tools for monitoring structural health and damage detection in materials and structures [18],[23]-[30].

\section{Acknowledgements}

Support for this work was received from the Italian Ministry of Foreign Affairs, Grant No. 00173/2014, Italy-USA Scientific and Technological Cooperation 2014-2015 ('Lavoro realizzato con il contributo del Ministero degli Affari Esteri, Direzione Generale per la Promozione del Sistema Paese'). 

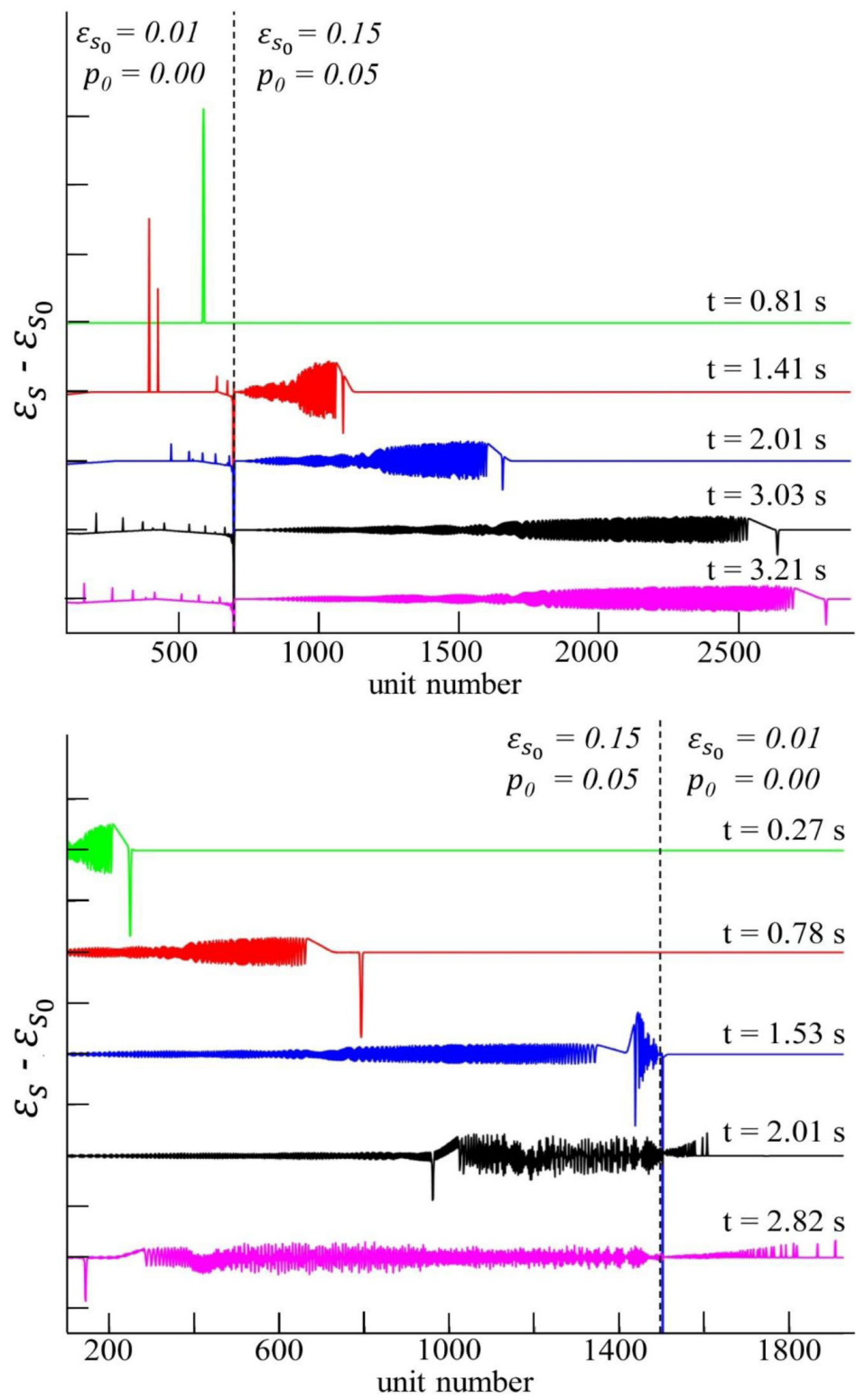

Figure 6: Top: Interaction of a rarefaction solitary wave with the interface between LIEH (left) and HIES (right) chains of tensegrity prisms. Bottom: Interaction of a rarefaction solitary wave with the interface between HIES (left) and LIEH (right) chains of tensegrity prisms.

\section{REFERENCES}

[1] T. Bückmann, M. Thiel, M. Kadic, R. Schittny, M. Wegener, An elastomechanical unfeelability cloak made of pentamode metamaterials. Nature Communications, 5, 4130, 2014.

[2] M. Maldovan, Sound and heat revolution in phononics. Nature, 503, 209-217, 2013.

[3] L. R. Meza, S. Das, J. R. Greer, Strong, lightweight, and recoverable three-dimensional ceramic nanolattices. Science, 345, 1322-1326, 2014. 
[4] W. van Grunsven, E. Hernandez-Nava, G.C. Reilly, R. Goodall, Fabrication and mechanical characterisation of titanium lattices with graded porosity. Metals, 4(3), 401-409, 2014.

[5] X. Zheng, J. DeOtte, M. P. Alonso, G. R. Farquar, T. H. Weisgraber, S. Gemberling, H. Lee, N. Fang, C. M. Spadaccini, Design and optimization of a light-emitting diode projection micro-stereolithography three-dimensional manufacturing system. Review of Scientific Instruments, 83, 125001, 2012.

[6] E. Hernández-Nava, C. J. Smith, F. Derguti, S. Tammas-Williams, F. Léonar, P. J. Withers, I. Todd, R. Goodall, The effect of density and feature size on mechanical properties of isostructural metallic foams produced by additive manufacturing. Acta Materialia, 85, 387-395, 2015.

[7] C. J. Smith, F. Derguti, E. Hernandez-Nava, S. Tammas-Williams, S. Gulizia, D. Fraser, I. Todd, Dimensional accuracy of Electron Beam Melting (EBM) Additive Manufacture with regard to weight optimized truss structures. Journal of Materials Processing Technology (in press).

[8] L. Yang, O. Harrysson, H. West, D. Cormier, Compressive properties of Ti6Al4V auxetic mesh structures made by electron beam melting. Acta Materialia, 60, 3370-3379,2012.

[9] R. E. Skelton, M. C. de Oliveira, Tensegrity Systems. Springer, 2010c.

[10] A. Amendola, F. Fraternali, G. Carpentieri, M. de Oliveira, R. E. Skelton, Experimental investigation of the softening-hardening response of tensegrity prisms under compressive loading. Composite Structures, 117, 234-243, 2014.

[11] F. Fraternali, G. Carpentieri, A. Amendola, On the mechanical modeling of the extreme softening/hardening response of axially loaded tensegrity prisms. Journal of the Mechanics and Physics of Solids,74, 136-157, 2015.

[12] M. Kadic, D. Bückmann, N. Stenger, M. Thiel, M. Wegner, On the practicability of pentamode mechanical metamaterials. Applied Physics Letters,100, 191901, 2012.

[13] ASTM E8 - 13a. Standard Test Methods for Tension Testing of Metallic Materials, DOI 10.1520/E0008 E0008M.

[14] L.E. Murr, S.M. Gaytan, F. Medina, E. Martinez, J.L. Martinez, D.H. Hernandez, B.I. Machado, D.A. Ramirez, R.B. Wicker, Characterization of Ti6Al4V open cellular foams fabricated by additive manufacturing using electron beam melting. Materials Science and Engineering A, 527, 1861-1868, 2010.

[15] I. Oppenheim, W. Williams, Geometric effects in an elastic tensegrity structure. Journal of Elasticity, 59, 51-65, 2000.

[16] V. F. Nesterenko, Dynamics of Heterogeneous Materials. Springer-Verlag, New York, 2001.

[17] C. Daraio, D. Ngo, V.F. Nesterenko, F. Fraternali, Highly nonlinear pulse splitting and recombination in a two-dimensional granular network. Physical Review E, 82, 036603, 2010. 
[18] F. Fraternali, L. Senatore, C. Daraio, Solitary waves on tensegrity lattices. Journal of the Mechanics and Physics of Solids, 60, 1137-1144, 2012.

[19] F. Fraternali, G. Carpentieri, A. Amendola, R. E. Skelton, V. F. Nesterenko, Multiscale tunability of solitary wave dynamics in tensegrity metamaterials. Applied Physics Letters, 105, 201903, 2014b.

[20] G. Theocharis, N. Boechler, C. Daraio, Nonlinear phononic structures and metamaterials. P.A. Deymier (ed.) Acoustic Matematerials and Phononic Crystals, Springer Series in Solid State Sciences, 173, 217-251, 2013.

[21] H. Lee, J. Zhang, H. Jiang, N. X. Fang, Prescribed pattern transformation in swelling gel tubes by elastic instability. Phys. Rev. Lett., 108, 214304, 2012.

[22] F. Fraternali, Free Discontinuity Finite Element Models in Two-Dimensions for In-Plane Crack Problems. Theoretical and Applied Fracture Mechanics, 47, 274-282, 2007.

[23] J. R. Raney, F. Fraternali, A. Amendola, C. Daraio, Modeling and in situ identification of material parameters for layered structures based on carbon nanotube arrays. Composite Structures, 93, (11):3013-3018, 2011.

[24] M. Modano, F. Fabbrocino, A. Gesualdo, G. Matrone, I. Farina, F. Fraternali, On the forced vibration test by vibrodyne. CompDyn 2015, 25-27 May 2015.

[25] T. El Sayed, W. Mock, A. Mota, F. Fraternali, M. Ortiz, Computational assessment of ballistic impact on a high strength structural steel/polyurea composite plate. Computational Mechanics, 43(4), 525-534, 2009.

[26] C. Donahue, P. Anzel, L. Bonanomi, T. Keller, C. Daraio, Experimental realization of a nonlinear acoustic lens with a tunable focus. Applied Physics Letters, 104, 014103, 2014.

[27] F. Fraternali, C.D Lorenz, G. Marcelli, On the estimation of the curvatures and bending rigidity of membrane networks via a local maximum-entropy approach. Journal of Computational Physics, 231, 528-540, 2012.

[28] B. Schmidt, F. Fraternali, Universal formulae for the limiting elastic energy of membrane networks. Journal of the Mechanics and Physics of Solids, 60, 172-180, 2012.

[29] F. Fraternali, I. Farina, G. Carpentieri. A discrete-to-continuum approach to the curvatures of membrane networks and parametric surfaces. Mechanics Research Communications, 56, 18-15, 2014.

[30] X. Ni, Nondestructive Evaluation And Structural Health Monitoring Based On Highly Nonlinear Solitary Waves. Phd Dissertation, University of Pittsburgh, USA, 2012. 